EPOS, XIII (1997), págs. 315-327

\title{
ALTERNATIVAS AL CONCEPTO CLÁSICO DE ENGLISHNESS EN LA LITERATURA CONTEMPORÁNEA
}

María Antonia Álvarez

UNED

\section{RESUMEN}

La literatura inglesa ha conservado durante siglos el concepto de Englishness con lo que supone de cultura, inteligencia y sensibilidad. A partir de la II Guerra Mundial se efectúan muchos cambios y se encuentran otras alternativas, aunque esta transferencia se basa principalmente en elementos procedentes del siglo XIX, pues lo que Englishness ha llegado a significar está fuera de todo patriotismo intolerante o política nacionalista e imperialista. Sin duda, el poder de los grandes novelistas ingleses del pasado ha sido tan fuerte como para imponer sobre los escritores y los lectores ingleses contemporáneos ciertas formas de mirar e interpretar la experiencia. Es como si Fielding, Jane Austen, Dickens o George Eliot se hubieran convertido en gigantescas presencias invisibles que obligaran a los ingleses a observar y valorar ciertas combinaciones de caracteres y situaciones, o como si trataran de desempeñar los papeles que les han adjudicado estas presencias invisibles.

Son muchos los escritores que se han ocupado del origen, desarrollo, fines o destino de Inglaterra. Según John Lucas (1990:I), el año 1688 marca el 
comienzo como nación moderna, sintiendo ya los escritores de esa época una especial responsabilidad por identificar el carácter nacional y buscar las características esenciales que los ingleses tenían en común.

Es posible analizar cómo artistas incluso aparentemente opuestos -Gainsborough y Reynolds o Constable y Turner- participan, no obstante, de su entomo, de manera que se descubre lo que tienen en común como ingleses, aunque pertenezcan a diferentes períodos. Ello se debe - según Nikolaus Pevsner (1955:3) - a ciertas peculiaridades que definen el concepto de Englishness: el gusto por lo perpendicular y lo horizontal, y el rechazo por el barroco, es una característica que rige todo el arte inglés; la arquitectura basada en compartimientos cuadrangulares o rectangulares es totalmente inglesa, propia de las iglesias anglosajonas. En la clásica casa de campo del siglo XVIII, el edificio también es cuadrangular y está formado tan sólo por líneas verticales y horizontales. Sin embargo, el pintoresco jardín que la rodea está diseñado con lagos irregulares, sendas retorcidas, que indican ese interés de los ingleses por la naturaleza y también por la tolerancia: a los árboles hay que dejarles que crezcan libremente y a los lagos que conserven su forma natural.

Que el jardín inglés participe de las teorías estéticas y políticas tiene otras implicaciones que van mucho más lejos. Raymond Williams se refiere en The Country and the City (1973:231) a las convenciones establecidas en torno a la casa de campo inglesa, asociada a la simplicidad, inocencia y libertad del campo, frente a la complejidad, corrupción y enclaustramiento de la ciudad; aunque estas mansiones puede que hayan sido también lugares de explotación. Una reminiscencia de ellas es la casa de campo donde va toda la familia para olvidarse de la ciudad durante el fin de semana, y sobre la que descarga toda su ironía Fay Weldon en su novela corta "Weekend»:

By seven-thirty they were ready to go. Martha had everything packed into the car and the three children appropriately dressed, and in the back seat, complete with educational games and wholewheat biscuits. When everything was ready in the car Martin would switch off the television, come downstairs, lock up the house, front and back, and take the wheel.

Weekend! Only two hours' drive down to the cottage on Friday evenings: three hours' drive back on Sunday nights. The pleasures of greenery and guests in between. They reckoned themselves fortunate, how fortunate! (p. 309) ${ }^{1}$

- A las siete y media estaban listos para salir. Martha había empaquetado todo en el coche y los tres niños adecuadamente vestidos en el asiento trasero, llenos de juegos educativos y galletas 
Según George Watson (1991:9), en Inglaterra este sentido de evadirse y recluirse en algún lugar recóndito tiene sus raíces en la insularidad, como si sus costas océanicas fueran de alguna manera intrínsecas a esta condición y esenciales a su forma de ser. Esto, que ha servido para vigilar su propia seguridad, tanto física como espiritual, también le ha hecho volverse hacía sí misma y esperar -en vez de acercarse ella a Europa- a que vayan los demás, aunque ya haya pasado la época imperial.

No obstante, el papel que desempeña la casa de campo en nuestro relato se aleja del concepto clásico de Englishness y sirve un fin distinto, ya que trata de resaltar la separación del individuo de su entomo familiar, siendo este aislamiento a la vez causa de resentimiento y frustración, pues Martha - la protagonista de «Weekend»- se encuentra separada del resto de su familia por ser ella la única que ha de realizar todo el trabajo del hogar mientras los demás se divierten:

On Fridays Martha would get home on the bus at six-twelve and prepare tea and sandwiches for the family: then she would strip four beds and put the sheets and quilt covers in the washing machine for Monday: take the country bedding from the airing basket, plus the books and the games, plus the weekend food-adquired at intervals throughout the week, to lessen the load... plus hairbrushes, jeans, spare T-shirts, Jolyon's antibiotics (he suffered from sore throats), Jenny's recorder, Jasper's cassette player and so on-ah, the so on!- -and would pack them all, skilfully and quickly, into the boot... Then Martha would run round the house tidying the wiping, doing this and that, finding the cat at one neighbour's and delivering it to another, while the others ate their tea; and would usually, proudly, have everything finished by the time they had eaten their fill. Martin would just catch the $\mathrm{BBC}_{2}$ news, while Martha cleared away the tea table... (p. 309) ${ }^{2}$

de trigo integral. Cuando todo estaba listo en el coche, Martin apagaba la television, bajaba las escaleras, cerraba la casa, la puerta principal y la de atrás, y cogía el volante.

¡Fin de semana! Sólo dos horas de coche para bajar al chalet los viernes por la tarde: tres horas a la vueita los domingos por la noche. Los placeres de la vegetación y de los invitados en medio. Se consideraban afortunados, jqué afortunados!

${ }^{2}$ Los viemes Martha llegaba a casa en autobús a las seis y doce y preparaba el té y los sandwiches para la familia: luego recogfa las cuatro camas y ponía las sábanas y la cubierta de los edredones en la lavadora para el lunes; cogía la ropa de cama del campo de cesta de la plancha, más los libros de juegos, más la comida del fin de semana-comprada a intervalos durante toda la semana, para reducir la carga-, los cepillos para el pelo, los vaqueros, las camisetas de repuesto, los antibióticos de Jolyon (padecía de dolor de garganta), la grabadora de Jenny, la cassette de Jasper, etc. —ah, iy qué etc.! - y lo empaquetaba todo, hábil y rápidamente en el portaequipajes... 
Este tema de encontrar un lugar en el campo para vivir aislado lo desarrolla John Fowles en Daniel Martin, donde expresa el sentimiento del protagonista de que ser inglés encarna el mito de Robin Hood, que consiste en retirarse a un lugar seguro y secreto con la musa femenina que inspire su arte. Si el héroe vuelve a casa finalmente es para conocer lo esencial de sí mismo, de su arte y de su país. Posteriormente, al igual que su héroe, Fowles explica lo que supone ser inglés en un ensayo que titula «On Being English But Not British" (1964). Empieza diferenciando la Gran Bretaña rojo-blanco-azul -el legado del mundo victoriano que concluyó finalmente con la II Guerra Mundialde la Inglaterra verde. Para esta última, el estado mental apropriado es ese concepto arquetípico muy primitivo, aunque aún presente, del justo proscrito, expresado en el mito de Robin Hood: ésta es la única leyenda nacional que puede afirmarse conocen todos los ingleses desde el comienzo del siglo $\mathrm{XV}, \mathrm{y}$ consiste esencialmente en una oposición crítica. Lo esencial de Robin Hood es estar en la oposición, no en el poder. A esta actitud se han adherido los grandes artistas ingleses, desde William Hogarth a Francis Bacon, y requiere no sólo un hábito mental y emocional de retiro, sino también de protesta, de disconformidad, junto a un estilo radical de vida. Los años sesenta quizá sean los menos Crusoe de la postguerra, pues la influencia extranjera -como lo demuestran los recursos novelísticos que adoman The French Lieutenant's Woman (1969), de John Fowles-, se consideraba de buen tono, aunque es significativo que esta novela de Fowles siga profundamente enraizada en una tradición realista.

La década de 1970 fue testigo de la vuelta a una disposición más confiada a escala nacional, con Kingsley Amis totalmente establecido como novelista y Philip Larkin como poeta - ambos desdeñosos de las novedades extranjeras-y con la nueva obra dramática de Tom Stoppard que no está de acuerdo con tomar prestados los valores del exterior.

En los años ochenta se produce un mayor rechazo de las modas importadas. Los novelistas y dramaturgos británicos escriben sobre su propio entorno, confiados en que también el mundo en general les seguía prestando atención, lo que representa un gran cambio, después de la época precedente, los años de entre guerras, claramente antibritánicos, con sus más distinguidos poetas —l americano T.S. Eliot y el irlandés W.B. Yeats-y sus

Luego Martha daba una vuelta a la casa ordenando y limpiando, haciendo esto y aquello, buscando al gato donde un vecino y dándoselo a otro, mientras los demás tomaban el té; y normalmente podia enorgullecerse de haber terminado todo para cuando ellos ya hablan comido lo suficiente. Martin conectaba las noticias de la $\mathrm{BBC}_{2}$, mientras Martha recogía la mesa del té... 
más grandes novelistas, aparte del grupo de Bloomsbury, como, por ejemplo, James Joyce. Por tanto, aunque la literatura inglesa dejara brevemente de ser británica, ocurre lo que parecía irreversible, y de nuevo se vuelve insular.

La tercera novela de Kingsley Amis se llama simbolicamente I Like It Here (1958). Otra que se publica ese mismo año, The Contenders, de John Wain, termina con una frase que resalta un término muy familiar: "en inglés decimos ho$m e^{\prime}$. Cuando a Philip Larkin le pregunta un periodista si alguna vez leía poesía extranjera, replica '¿poesía extranjera?'. La primera novela de John Braine, Room at the Top (1957), se sitúa en su ciudad natal, en Yorkshire. Y Irish Murdoch normalmente escribe como una londinense que está orgullosa de serlo. En A Word Child (1975), como son tan específicas sus referencias a ciertos lugares, el narrador comenta que a veces estaba decidido a llamar a su historia $E l$ Círculo Interior; y London Fields (1989), de Martin Amis, describe de forma tan detallada Notting Hill como si fuera una guía. Todo esto supone una nueva imagen literaria centrada exclusivamente en lo inglés, aunque se hayan buscado nuevas alternativas al concepto clásico.

Las novelas londinenses de Irish Murdoch -según Louis L. Martz (1971:69) tienen la cualidad dickensiana del detalle, porque tienen su origen en un profundo afecto instintivo hacia el marco de Londres, tanto si es sórdido y desarrapado como elegante. Sin embargo, en su novela, Bruno's Dream, Murdoch vuelve a la búsqueda del yo, la búsqueda de identidad por parte del héroe, a fin de poder comprender quién es y cúal es su función dentro de su entorno. En toda la literatura inglesa de postguerra se presenta al hombre contemporáneo como falto de identidad o, al menos, inquieto por conocerse a sí mismo. Spiering (1992:4-5) ha estudiado ampliamente el concepto de Englishness y afirma que en la crítica cultural y literaria de esta época la búsqueda de identidad nacional juega un papel central. Un buen ejemplo de ello es la heroína de «Weekend», quien se cuestiona continuamente el papel que tiene que desempeñar en su entorno familiar:

If Martha chose to go out to work - as was her perfect rightMartin allowed, even though it wasn't the best thing for the children, but that must be Martha's moral responsibility. (p. 310) ${ }^{3}$

${ }^{3}$ Si Martha eligió salir a trabajar - a lo que tenía perfecto derecho- Martin se lo permitra, aunque no fuera lo mejor para los niffos; pero eso debra ser la responsabilidad moral de Martha. 
Dentro de esta búsqueda de identidad aparece la preocupación predominante de la ficción inglesa a través de toda su historia por el concepto de clase, que surge en cualquier parte y época de la novela. El esnobismo y la aspiración personal —según Walter Allen (1964:2) - inseparables de la noción de clase social han sido una fuente para lo cómico en la ficción inglesa. Los americanos son mucho menos conscientes de la actitud clasista que los ingleses, quienes están obsesionados con ella hasta un grado casi patológico. En «Weekend», Martha compara la vida que había llevado su madre con la suya, siempre expresándolo con ese fuerte sentido irónico de la autora:

Martha's mother had led a secluded suspicious life, and made Martha's childhood a chilly and a lonely time. Life now, by comparison, was wonderful for Martha. People, children, houses, conversations, food, drink, theatres-even, now, a career. (p. 311) ${ }^{4}$

Además del concepto de clase, más acentuado en la época victoriana, la década de 1950 redescubrió el campo de batalla del sexo: una batalla que, aunque familiar para la época anterior, había sido generalmente abandonada como tema literario en los años de entre guerras. La nueva oleada comenz6 en 1968, en Nueva York, y fue pronto conocida como the Women's Movement. No obstante, los escritores habían trazado sus líneas de batalla y abierto sus ataques: Look Back in Anger (1956), de John Osborne, es una obra dramática radical, con diatribas antifeministas. (En Mantissa (1982), de John Fowles, el héroe termina diciendo que las mujeres habían inventado la literatura para vengarse de los hombres, y Stanley and the Women (1984), de Kingsley Amis, es una novela furiosamente masculina). La nueva oleada de feminismo de finales de los sesenta, con Female Eunuch (1970), de Germaine Greer, renovó la declaración de guerra. Es una literatura obsesionada por la aceptación social, pues las feministas de la década de 1960 creían que los hombres tomarían más en serio a las mujeres si eran profesionales, lo que parece ser la preocupación de la protagonista de «Weekend»:

Ah, she was grateful: little earnest Martha, with her shy ways and her penchant for passing boring exams-how her life had blossomed out! (p. 311)

4 La madre de Martha habra llevado una vida recelosa y recluída, y convirtio la niffez de Martha en una Epoca fría y solitaria. Ahora la vida, por comparación, era maravillosa para Martha. Gente, hijos, casas, conversaciones, comida, bebida, teatros; incluso, ahora, una profesion. 
Ah, to be all things to all people: children, husband, employer, friends! It can be done: yes, it can: super woman. (p. 312) ${ }^{5}$

Pero ahora Marta no es capaz de simultanear sus obligaciones como ama de casa y el trabajo en la oficina, y por ello trata de encontrar la forma de mantener su puesto de investigadora de mercados en una agencia publicitaria sin dejar de responder a las fuertes demandas de su marido:

Martin can't bear bad temper. Martin likes slim ladies. Diet. Martin rather likes his secretary. Diet. Martin admires slim legs and big bosoms. How to achieve them both? Impossible. But try, oh try, to be what you oght to be, not what you are. Inside and out. (p. 312) ${ }^{6}$

Por tanto, esta novela corta de Fay Weldon, puede clasificarse claramente dentro de la literatura feminista, ya que se trata de un libro de denuncia y protesta de las mujeres que tienen que realizar en la vida el doble papel de esposas y madres al mismo tiempo que trabajan fuera del hogar:

Cook! Ah, cook. People love to come to Martin and Martha's dinners. Work it out in your head in the lunch-hour. If you get in at six-twelve, you can seal the meat while you beat the egg while you feed the cat while you lay the table while you string the beans while you set out the cheese... $\longrightarrow$ oh, bed, sleep, peace, quiet. (p. 313) ${ }^{7}$

Entre los temas principales de la literatura feminista, están la necesidad de compromiso y relaciones sociales, pero también el sentirse sexualmente realizada:

s ¡Estaba tan agradecida! La pequeña y formal Martha, con su timidez e interés en aprobar exámenes aburridos... ¡Cómo se habla transformado su vida! woman.

¡Ser todo para los demás: hijos, marido, jefe, amigos! Puede hacerse; st, se puede: la super-

- Martin no puede soportar el mal humor. A Martin le gustan las señoras delgadas. Dieta. A Martin le gusta más bien su secretaria. Dieta. Martin admira las piemas delgadas y los pechos abultados. ¿Cómo conseguir ambas cosas? Imposible. Pero intenta, intenta ser lo que tienes que ser, no lo que eres. Por dentro y por fuera.

${ }^{7}$ COcinar! ¡Ah, cocinar! A la gente le gusta venir a las cenas de Martin y Martha. Organizarlo en tu cabeza a la hora del almuerzo. Si estás de vuelta a las seis y doce, puedes acabar de dorar la carne mientras bates el huevo a punto de nieve, mientras das de comer al gato, mientras pones la mesa, mientras quitas las hebras a los guisantes, mientras arreglas el queso... ¡Oh! la cama, dormir, paz, silencio. 
Sex! Ah, sex... you don't want his secretary providing a passion you neglected to develop. Do you? Quick, quick, the cosmic bond. Love. Married love. (p. 313) ${ }^{8}$

Todo el problema parece radicar en la vida familiar, que ha dejado de ofrecer la estabilidad necesaria para que puedan desarrollarse plenamente los miembros que la componen. Y eso es lo que Martha, la heroína de Fay Weldon, trata lealmente de conseguir con su constante esfuerzo:

Then supper - pork chops in sweet and sour sauce ('Pork is such a dull meat if you don't cook it properly': Martin), green salad from the garden, or such green salad as the rabbits had left ('Martha, did you really net them properly? Be honest now!': Martin) and sauté potatoes. Mash is so stodgy and ordinary, and instant mash unthinkable. (p. 314) ${ }^{9}$

Para transferir su mensaje al lector, la autora presenta dos clases de mujeres diferentes: la mujer egoista y la mujer sacrificada. Janet, la primera esposa de Colin, el mejor amigo de la familia, y también Martha, el personaje principal del relato, representan el tipo femenino de esposa sacrificada por su hogar:

Janet was rather like Martha, quieter and duller than her husband. A nag and a drag, Martin rather thought, and said, and of course she'd let herself go, everyone agreed. No one exactly excused Colin for walking out, but you could see the temptation. (p. 315) ${ }^{10}$

Y Katie —que ha sucedido a Janet— es el típico ejemplo de una mujer superficial, egoísta:

Katie was languid, beautiful and elegant. She drawled when she spoke. Her hands were expressive: her feet were like a female. She had no children. (p. 315)"

- ¡Sexo! Ah, el sexo... ¿no querrás que su secretaria le proporcione una pasión que tú has descuidado avivar, verdad? Rápido, rápido, el vínculo cósmico. Amor. El amor marital.

- Luego la cena: chuletas de cerdo con salsa agridulce («el cerdo es una comida tan insípida si no la guisas debidamenten: Martin), verduras del jardín para la ensalada, o la ensalada que han dejado los conejos ( $\ll$ Martha, ¿de verdad los metiste en la red como es debido? jdi la verdad! : Martin) y patatas salteadas. El puré de patatas es tan pesado y ordinario, y el que ya está preparado es inconcebible.

10 Janet era parecida a Martha, más callada y aburrida que su marido. Gruñona y pesada, pensaba Martin, y lo decía; todos estaban de acuerdo en que se había abandonado. Realmente nadie excusaba a Colin por haberse ido, pero podían comprender la tentación.

${ }^{11}$ Katie era lánguida, bella y elegante. 1 blaba de manera pausada. Sus manos eran expresivas; sus pies, femeninos. No tenía hijos. " 
Así pues, Fay Weldon presenta los dos tipos de mujer, contrastando las cualidades de ambas; mientras la protagonista del relato dedica todas las horas del día a organizar la vida familiar:

Martha knew that if breakfast for seven is to be manageable the sink must be cleared of dishes. A tricky meal, breakfast. Especially if bacon, eggs, and tomatoes must all be cooked in separate pans. ('Separate pans mean separate flavours!': Martin) (p. 316) ${ }^{12}$

La otra sólo piensa en lucirse, sin importarle nada de lo que la rodea:

Katie weeded with rubber gloves on and pulled out pansies in mistake for weeds and laughed and laughed along with everyone when her mistake was pointed out to her, but the pansies died. (p. 316) ${ }^{13}$

Sin embargo, entre los dos tipos de mujer presentados por Fay Weldon, la triunfadora no es la madre y esposa sacrificada, sino la mujer egoísta, que consigue brillar por encima de la otra, aunque no hay que olvidar el fuerte sentido irónico:

'It does one no good to be materialistic', Katie confided, 'I have nothing. No home no family, no ties, no possessions. Look at me! Only me and a suitcase of clothes.' But Katie seemed highly satisfied with the me and the clothes were stupendous. Katie drank a great deal and became funny. Everybody laughed, including Martha. Katie had been married twice. Martha marvelled at how someone could arrive in their mid-thirties with nothing at all to their name, neither husband, nor children, nor property and not mind. Mind you, Martha could see the power of such helplessness. If Colin was all Katie had in the world, how could Colin abandon her? And to what? Where would she go? How would she live? Oh, clever Katie. (p. 318) ${ }^{14}$

12 Martha sabía que si se quiere que el desayuno para los siete sea manejable, el fregadero tiene que estar vacio de vajilla. Una comida complicada, el desayuno. Especialmente si el bacon, los huevos y los tomates tienen que cocinarse todos en distintas sartenes. ( $i D i s t i n t a s$ sartenes significan sabores separados!': Martin)

${ }^{13}$ Katie quitaba las hierbas con los guantes de goma puestos y arrancaba los pensamientos en vez de la hierba, y se ref́a con todos cuando la seffalaban su equivocación, pero los pensamientos se morfan.

14 -No nos hace ningún bien ser materialistas, -confeso Katie-. Yo no tengo nada. Ni casa ni familia, ni ataduras, ni posesiones. Mírame! S6́lo yo y una maleta de ropa. - Pero Katie parecía muy satisfecha con el yo y las ropas eran estupendas. Katie bebía mucho y era muy graciosa. Todos se refan, incluso Martha. Katie se había casado dos veces. Martha se maravillaba de cómo 
Martha, por el contrario, tiene que seguir preocupándose para que todo funcione en el hogar:

So Martha perched uneasily on the steps and had a glass of cider, and wondered how, if lunch was going to be late, she would get cleared up. (p. 319) ${ }^{15}$

Ya hemos dicho que un elemento importante para el concepto de Englishness es lo rural - presente en la mayoría de las obras de la literatura inglesa posteriores a la II Guerra Mundial-, y que una reminiscencia de la vida rural son los jardines ingleses que no pueden separarse del concepto de hogar inglés. Aquí Fay Weldon nos habla de las famosas rosas inglesas, pero siempre desde el punto de vista de la heroína y narradora, que todo lo ve como un cúmulo de tareas que ella sola tiene que realizar:

Real roses round the door.

Roses. Prune, weed, spray, feed, pick. Avoid thorns. One of Martin's few harsh words. (p. 313) ${ }^{16}$

Y también Martin utiliza el tema de las rosas con un cierto tono de burla hacia su mujer; éste es un elemento típico de Englishness, expresar algún juicio de valores con sentido del humor: el típico humor inglés.
'Martha, you can't want roses! What kind of person am I married to? An anti-rose personality?' (p. 304) ${ }^{17}$

Este tema rural se presenta con el contraste campo-ciudad -aquí representado por los dos tipos femeninos- donde inmoralidad y egoísmo se identifican con los defectos intrínsecamente urbanos. Según Spiercing (1992:179), la visión de Inglaterra como una comunidad esencialmente rural cristalizó a fina-

alguien podía llegar a la mitad de los treinta sin nada en absoluto que le perteneciera, ni marido, ni hijos, ni propiedad y que no le importara. Hay que confesar que Martha comprendía el poder de tal desamparo. Si Colin era todo lo que Katie tenía en el mundo, ¿cómo podría abandonarla? ¿Y para qué? ¿Dónde iría? ¿Cómo podría vivir? Oh, inteligente Katie.

is Por ello Martha se sentó intranquila en las escaleras y se tomó un vaso de sidra, preguntándose cómo, si la comida iba a ser tan tarde, podría limpiarlo todo.

${ }^{16}$ Rosas naturales alrededor de la puerta.

Rosas. Poda, siembra, riega, abona, recoge. Evita las espinas. Una de las pocas palabras ásperas de Martin.

17 -Martha, ¡no puede ser que no quieras rosas! ¿Con que clase de persona me he casado? ¿Una personalidad anti-rosas? 
les del siglo XIX y ha seguido siendo desde entonces una de las imágenes centrales de Englishness. En «Weekend» aparece esta imagen, pero muy alejada de su concepto clásico:

Green grass. Oh, God, grass. Grass must be mown. Restful lawns, daisies bobbing, buttercups glowing. Roses and grass... (p. 313) ${ }^{18}$

El tema rural transmite una imagen positiva, donde Englishness es igual a humanidad. Esa idea de que el yo es más humano que el otro ha desempeñado siempre un papel importante al tratar de concretar la imagen de identidad nacional. Para Spiering (1992:179), aunque no se considere lo rural como un coto exclusivamente inglés, la idea de que el yo nacional está enraizado en el campo y haya que protegerle de los focos de influencia exterior, aparece como algo predominante en la Inglaterra de postguerra. Orwell reconoce el valor de los recuerdos de un estilo de vida más simple cuando escribe: «el hombre es un ser humano sólo si consigue seguir siendo simple, a pesar de la tendencia exterior a debilitar su conciencia, a embotar su curiosidad.» Para Orwell, «fuera de mi trabajo lo que más me importa es cuidar mi jardin, especialmente cultivar vegetales» (1975:110); así pues, bajo el sentido de nostalgia está la asociación no sólo con una vida más simple, sino también con la idea pastoral romántica.

En esa lucha que se plantea la heroína por conseguir llegar a ser la mujer ideal o modelo de mujer nueva -lo que Fay Welson denomina super-woman- intervienen dos elementos principales, la amabilidad y la delicadeza, que figuran de forma destacada en la valoración femenina de Englishness. Si ser inglés supone poseer una cualidad humana especial, pertener a una clase determinada de criaturas, en «Weekend», ser amable y delicada es un punto clave para tener éxito:

'Don't fret, Martha. I'll do it.' 'Don't fret.' Martha clearly hadn't been smiling enough. She was in danger, Martin implied, of ruining everyone's weekend. Martin frowned at Martha: he thought the appearance of martyrdom in the face of guests to be an unforgivable offence. (p. 323) ${ }^{19}$

18 Hierba verde. Oh, Señor, la hierba. Hierba que hay que cortar. Césped en reposo, margaritas agitándose, ranúnculos brillando. Rosas y hierba...

19 -No te apures, Martha. Yo lo hare. No te apures. Sin duda Martha no había estado bastante sonriente. Martin dio a entender que ella corria el riesgo de arruinar el fin de semana de todos. Martin miro con ceño a Martha, pensaba que aparecer como una mártir delante de los invitados era una ofensa imperdonable. 
Otros aspectos, como la comida inglesa, también tienen características especiales. En «Weekend» hay muchos ejemplos:

Saturday and Sunday lunch shone like reassuring beacons in their lives. Saturday lunch: family lunch: fish and chips. ('So much better cooked at home than bought': Martin) Sunday. Usually roast beef, potatoes, peas, apple pie. Oh, of course. Yorkshire pudding. (p. 319) ${ }^{20}$

En resumen, la literatura ha conservado durante siglos el concepto de $E n$ glishness, con lo que supone de cultura, inteligencia y sensibilidad. A partir de la II Guerra Mundial se efectúan muchos cambios y se encuentran otras alternativas, aunque esta transferencia, como sugiere Brian Doyle (1989:21), se basa principalmente en elementos procedentes del siglo XIX. Sólo como consecuencia del esfuerzo realizado por la literatura, la lingüística o la historia ha sido posible cuestionar las normas culturales y educacionales anteriores. Según Doyle (1989:40), lo que Englishness ha llegado a significar está fuera de todo patriotismo intolerante o política nacionalista e imperialista. Sin duda, el poder de los grandes novelistas ingleses del pasado ha sido tan fuerte como para imponer sobre los escritores y los lectores ingleses contemporáneos ciertas formas de mirar e interpretar la experiencia. Es como si Fielding, Jane Austen, Dickens o George Eliot se hubieran convertido en gigantescas presencias invisibles que obligaran a los ingleses a observar y valorar ciertas combinaciones de caracteres y situaciones, o como si trataran de desempeñar los papeles que les han adjudicado estas presencias invisibles.

Sin embargo, quizá la influencia de la lengua y la literatura inglesas nunca haya sido tan grande como en la segunda mitad del siglo XX. Como afirma George Watson (1991:2), después de 1945 la lengua inglesa se ha convertido en una lingua franca - la primera vez que la humanidad ha conocido un hecho semejante- y más de la mitad del correo mundial utiliza ahora el inglés. La importancia de su teatro ha sido generalmente reconocida desde mediados de los años cincuenta; al igual que la novela, traducida a todos los idiomas, no puede ignorarse, y su poesía ha mostrado vitalidad más que suficiente para brillar por sí misma, aunque en todos ellos la noción de Englishness haya cambiado de manera considerable, rechazando su significado original y olvidando su gran influencia espiritual.

20 Los almuerzos del sábado y del domingo brillaban como faros alentadores en sus vidas. Almuerzo del sábado: comida familiar: fish and chips. ( Mucho mejor hecho en casa que comprado»: Martin). Domingo. Roast beef, patatas, guisantes, tarta de manzana. Oh, desde luego. Yorkshire pudding. 


\section{REFERENCIAS BIBLIOGRÁFICAS}

AlLEN, WALTER (1964) Tradition and Dream: The English and American Novel from the Twenties to our Time. London: J.M. Dent \& Sons.

DOYLE, BRIAN (1989) English and Englishness. London and New York: Routledge.

GILPIN, GeORGE H. (1991) The Art of Contemporary English Culture. London: Macmillan.

INGLISH, FRED (1969) The Englishness of English Teaching. London \& Harlow: Longmans, Green and Co. Ltd.

Johnston, John H. (1984) The Poet and the City. Athens: The University of Georgia Press.

LEAVIS, F.R. 1974 (1948) The Great Tradition. Penguin Books.

LEAvIS, Q.D. (1983) Collected essays. Volume I.The Englishness of the English Novel. Cambridge Univ.Press.

LODGE, DAVID (1970) «Literary Criticism in England in the Twentieth Century», en Bergonzi, Bernard (ed.) The Twentieth Century. London: Barrie \& Jenkins, pp. $362-403$.

LuCAs, JOHN (1990) England and Englishness. London: The Hogarth Press.

Maes-JelineK, Hena (1970) Criticism of Society in the English Novel Between the Wars. Paris: Société d'Editions.

MaRTZ, Louis L. (1971) «Irish Murdoch: The London Novels», en Brower, Reuben A. (ed). Twentieth-Century Literature in Retrospect. Cambridge, Massachusetts: Harvard University Press.

McEwan, Nell (1987) Perspective in British Historical Fiction Today. London: Macmillan.

ORWELl, GeORge (1975) The Critical Heritage, en Jeffrey Meyers (ed.). London: Routledge and Kegan Paul.

PeVsner, Nikolaus (1955) The Englishness of English Art. BBC Reith Lectures.

SAGE, LORNA (1979) «Female Fictions: The Women Novelists», The Contemporary English Novel, en Malcolm Bradbury and David Palmer (eds.), Stratford-uponAvon Studies 18. London: Amold, pp. 80-87.

SPIERING, M. (1992) Englishness, Foreigners and Images of National Identity in Postwar Literature. Amsterdam: Rodopi.

STEINER, GEORGE (1967) «Literature and Post-History», en Language and Silence. New York: Atheneum.

WATSON, GeORGE (1991) British Literature since 1945. London: Macmillan.

WELDON, FAY (1987) «Weekend», en The Penguin Book of Modern British Short Stories, Malcolm Bradbury (ed.). London: Penguin Books.

WILlIAMS, RAYMOND 1987 (1985) The Country and the City in the Modern Novel. London: Hogarth. 